\title{
Immune checkpoint inhibitors for esophageal squamous cell carcinoma: a narrative review
}

\author{
Wencheng Zhang, Ping Wang, Qingsong Pang \\ Department of Radiation Oncology, Tianjin Medical University Cancer Institute and Hospital/National Clinical Research Center for Cancer, \\ Tianjin, China \\ Contributions: (I) Conception and design: Q Pang; (II) Administrative support: P Wang; (III) Provision of study materials or patients: W Zhang; (IV) \\ Collection and assembly of data: W Zhang; (V) Data analysis and interpretation: W Zhang; (VI) Manuscript writing: All authors; (VII) Final approval \\ of manuscript: All authors. \\ Correspondence to: Qingsong Pang; Ping Wang. Department of Radiation Oncology, Tianjin Medical University Cancer Institute and Hospital, \\ Huanhu West Road, Hexi District, Tianjin, China. Email: pangqingsong@tjmuch.com; wangping@tjmuch.com.
}

\begin{abstract}
Esophageal cancer (EC) has the seventh highest incidence and the sixth highest mortality rate of any type of cancer worldwide. In China, esophageal squamous cell carcinoma (ESCC) accounts for more than $95 \%$ of EC patients. The main treatment for EC patients is surgery and/or chemoradiotherapy (CRT). A large proportion of EC patients are already at an advanced stage of the disease by the time they are diagnosed. In these cases, CRT is left as the only treatment choice, and the treatment outcome is poor. Immune checkpoint inhibitors (ICIs) can improve clinical response and patient survival of patient with many types of tumors through reactivating antitumor immune response. The study of ICIs in ESCC is relative delayed compared with that in other solid tumors. Recent results from clinical trials have demonstrated the safety and efficacy of ICIs either alone or combined with chemotherapy or chemoradiotherapy in ESCC patients. Accumulated evidences also have shown the improved treatment outcome was associated with PDL1 expression, tumor DNA instability-induced tumor mutational burden (TMB), and drawing lymphocytes into the tumor. Based on these findings, ICIs combined with CRT or radiotherapy (RT) are the focus of ongoing studies. This review will summarize the recent progress in this field, especially the mechanism of ICIs used in ESCC, their clinical efficacy and toxicities, and potential biomarkers.
\end{abstract}

Keywords: Esophageal squamous cell carcinoma (ESCC); immune checkpoint inhibitor (ICI); immunotherapy; chemotherapy; radiotherapy (RT); biomarker

Submitted Apr 16, 2020. Accepted for publication Jul 27, 2020.

doi: $10.21037 /$ atm-20-4625

View this article at: http://dx.doi.org/10.21037/atm-20-4625

\section{Introduction}

In 2018, there were approximately 570,000 new cases of esophageal cancer (EC) and around 510,000 related deaths globally, placing it seventh and sixth among all cancer types in terms of morbidity and mortality, respectively (1). China accounts for half of EC cases worldwide. EC comprises two histological subtypes, esophageal adenocarcinoma (EAC) and esophageal squamous cell carcinoma (ESCC), which each have distinct pathological features and molecular characteristics (2). In contrast with Europe and the United
States, where EAC is more common, $95 \%$ of EC cases in China are ESCC. Surgery remains the mainstay for treatment of ESCC at early stage. Several randomized controlled trials demonstrated that preoperative chemoradiotherapy (CRT) compared with surgery alone increases the overall survival (OS), but the treatment-related adverse effects (TRAEs) raised either $(3,4)$. In China, $60 \%$ to $70 \%$ of patients have already developed locally advanced or advanced disease at the time of diagnosis (5). For these patients, concurrent CRT (CCRT) is the only choice. But the treatment outcome remains poor. Although the 

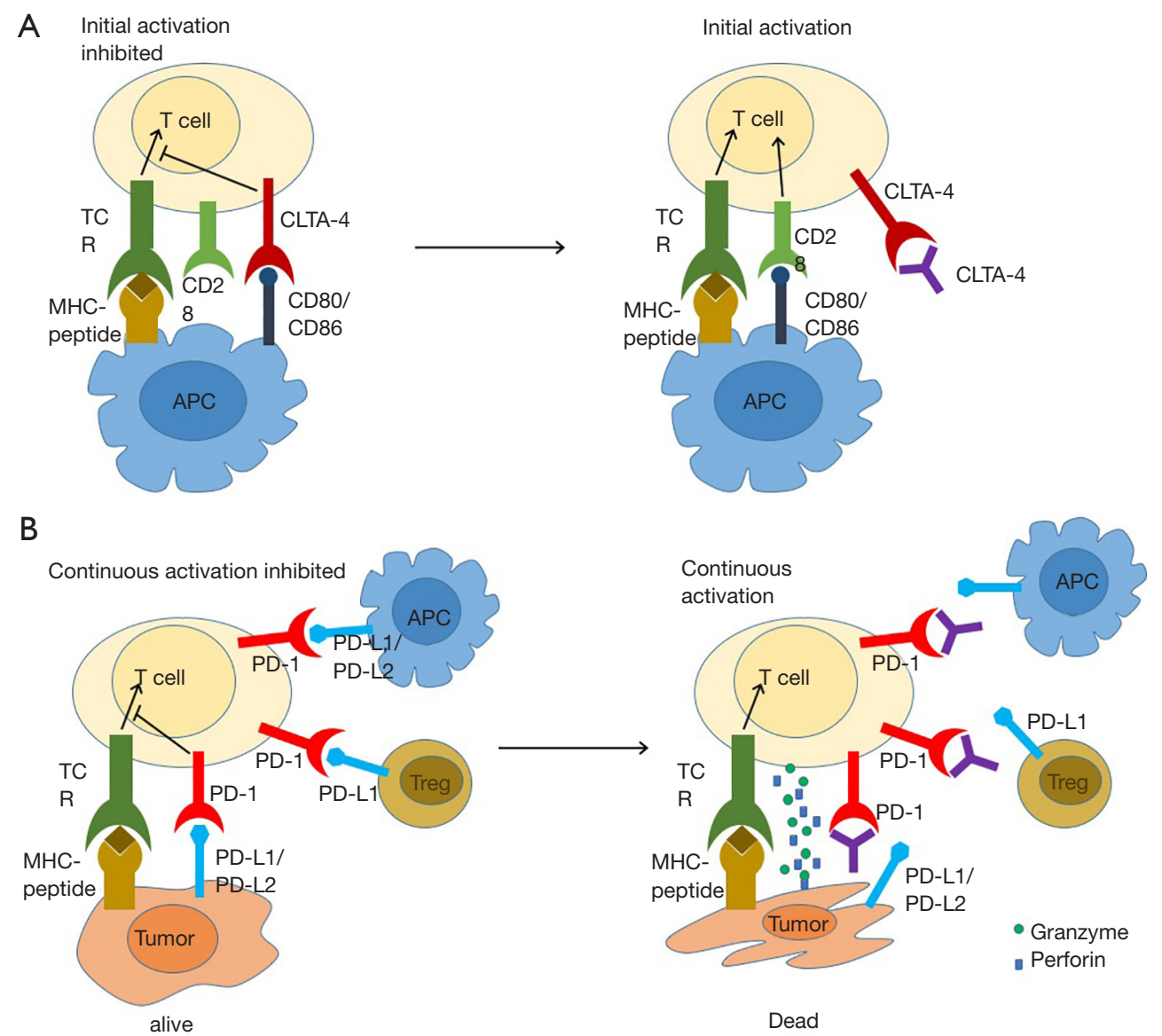

Figure $1 \mathrm{~T}$ cell inhibition and T cell activation by checkpoint inhibition. (A) The binding of CTLA-4 and CD80/CD86 that were expressed on antigen presenting cells blocked the downstream transduction of activation signaling resulting from the recognition of T-cell receptor and MHC-antigen complex. CTLA-4 inhibitors that targeted CTLA-4 prevented the binding of CTLA-4 from CD80/CD86 and restored the initial activation of $T$ cells. (B) The interaction of PD-1 on activated $T$ cells and its ligands PD-L1/PD-L2 on tumor cells, antigen presenting cells and regulatory $\mathrm{T}$ cells inhibited the continuous activation of $\mathrm{T}$ cells. PD-1/PD-L1 inhibitors that targeted the PD-1/PD$\mathrm{L} 1 / 2$ axes could maintain the continuous activation of $\mathrm{T}$ cells.

antibodies that target epidermal growth factor receptors have been investigated in ESCC, the results from clinical trials did not indicate positive results (6). As a result, new and effective strategies for advanced ESCC are urgently needed.

$\mathrm{T}$ cells plays an important role in immune defense against cancer. T-cell activation is strictly regulated by stimulatory and inhibitory ligand-receptor interaction between $\mathrm{T}$ cells, dendritic cells, macrophages and tumor cells in the tumor microenvironment. These ligandreceptor pairs that negatively regulates $\mathrm{T}$-cell activation are named "immune checkpoints". A growing number of studies have demonstrated that immune checkpoint inhibitors (ICIs), such as anti-CTLA-4 mAb (ipilimumab) and anti-PD1 mAb (nivolumab and pembrolizumab), can promote tumor shrinkage and improve overall survival in a variety of tumors (Figure 1). Since ipilimumab was approved firstly by FDA for the treatment of metastatic melanoma in 2011, additional ICIs that all target the PD-1/PD-L1 axis have been approved for the treatment of a broad range of tumor types. Research on ICIs in ESCC started later, while several ongoing clinical trials featuring anti-PD-1 mAb have shown promising results. The development of ICIs has been explored (7). This review focused on clinical application of ICIs for treating ESCC, including the feasibility and efficiency of mono or combined treatments of ICIs, the safety and TRAEs as well as biomarkers that were studied as hotspots in recent years. We present the following article in 


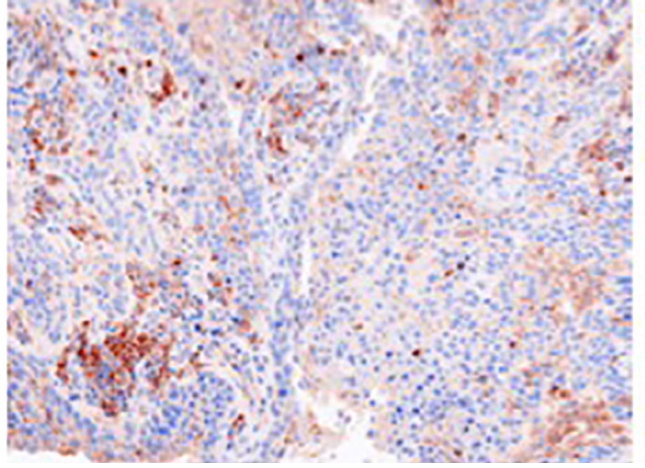

PD-1

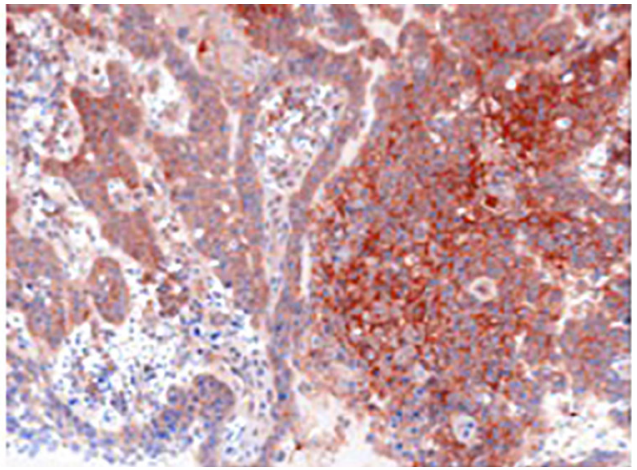

PDL1

Unpublished data

Figure 2 PD-1/PD-L1 expression in esophageal squamous carcinoma cell. PD-1 expressed on the membrane of tumor-infiltrating lymphocytes (A) and PD-L1 expressed on the membrane of tumor cells (B) were assessed by immunochemistry assay (200x).

accordance with the Narrative Review reporting checklist (available at http://dx.doi.org/10.21037/atm-20-4625).

\section{ICIs required for ESCC treatment}

Heterogeneous factors in the tumor microenvironment limit antitumor immunity and support tumor evasion. The function of antitumor effector $\mathrm{T}$ cells is inhibited by the interaction between checkpoint molecules and their ligands expressed on tumor cells, stromal cells, and immune cells, which ultimately leads to immune escape $(8,9)$. In ESCC, the checkpoint ligand PD-L1 has been investigated most extensively. PD-L1 expression on tumor cells is regulated by both intrinsic and exogenous factors (10) (Figure 2). Ohigashi et al., who evaluated PDL1 or PD-L2 gene expression in 18 (43.9\%) of 41 ESCC tumor samples using real-time PCR method, showed for the first time that PD-L1 was a novel prognostic marker for human EC (11). Meanwhile, in a meta-analysis involving 1,350 ESCC patients from China and Japan, PD-L1 overexpression was found in 559 patients (41.4\%) and indicated to be a poor prognostic biomarker for ESCC (12). Immunohistochemistry (IHC) was the only method for evaluating PD-L1 expression used in this study (12). Tsutsumi et al. also reported an association between PD-L1 expression and worse rates for OS and relapse-free survival (RFS) (13). Patients with positive PD-L1 expression have significantly lower pathological complete response rates ( $13 \%$ versus $32 \% ; \mathrm{P}=0.036)$ than those with negative PD-L1 expression. In addition, Huang et al. discovered that PD-L1 expression influenced the effect of neoadjuvant CRT in ESCC patients, finding PDL1 expression to be an independent predictor of poor CRT response and worse treatment outcome (14).

Tumor mutational burden (TMB) represents the number of genetic changes in a tumor. Tumors with a high TMB are likely to express high levels of neoantigens, and therefore could potentially possess high capability to attract $T$ cells that participate in antitumor immune response. Several studies have shown high TMB in ESCC $(15,16)$. Yarchoan et al. observed a significant correlation between TMB and the objective response rate (ORR) (17). Microsatellite instability (MSI) is caused by the failure of the DNA mismatch repair (MMR) system, which is usually responsible for repairing DNA. High MSI (MSI-H) typically correlates with increased neoantigen burden, resulting in a much greater response to ICIs. The antiPD-1 mAb pembrolizumab (Keytruda) is the first FDAapproved treatment based on MSI-H or MMR deficiency, regardless of cancer types. Studies on MSI have shown a much lower level of MSI-H in ESCC than in gastric cancer (2). The relatively high levels of PD-L1 expression and TMB in ESCC, as well as MSI-H in a proportion of patients, indicate that patients with ESCC might be susceptible to ICI treatment.

Tumor-infiltrating lymphocytes (TILs), which influence clinical response and survival, are important components in tumor microenvironment. With increased checkpoint expression and reduced antitumor function, $\mathrm{CD}^{+}$helper $\mathrm{T}$ cells and $\mathrm{CD} 8^{+}$effector $\mathrm{T}$ cells in tumors become exhausted in the inhibitory immune microenvironment. In contrast, inhibitory TILs, such as the regulatory T cells (Tregs) and 
tumor-associated macrophages (TAMs), are activated by the elevated production of IL10, IL6, transforming growth factor- $\beta$ (TGF- $\beta$ ), and expression of checkpoint molecules. Meanwhile, the density and location of TILs also affect their role in tumors. Tumors can be classified according to the characteristics of TILs into four categories: hot, alteredexcluded, altered-immunosuppressed, and cold. Hot tumors, which are also defined as inflamed tumors, have high levels of TILs. In altered-excluded tumors, TILs are excluded around the tumor margins, while in immunosuppressed tumors, TILs are either scattered in or surround the tumor. Owing to the immunosuppressive microenvironment, the number of TILs in immunosuppressed tumors is much lower than that in altered-excluded tumors. Finally, cold tumors, also known as non-inflamed tumors, are devoid of TILs. Although no study has identified tumor immune categories in EC, it has been reported that a high density of CD8 cells was significantly correlated with more favorable OS and RFS in patients who received postCRT (18). Similarly, we found that high levels of TILs induced by CCRT were associated with improved clinical response and survival (19). Furthermore, the effect of intratumoral location of TILs on the survival of ESCC patients varies. High numbers of CD8+ and PD-1+ TILs only in the surface and center of the tumors were related to improved OS (20). Optimal therapeutic strategies that target the different tumor categories should be provided. Of these four tumor categories, hot tumors may have higher susceptibility to PD-1/PD-L1 blockade. Meanwhile, CRT and RT combined with immunotherapy might be optimal for the other tumor categories in inducing and activating antitumor immune response and attracting more functional T cells into tumors.

\section{The use of ICls in EC (Table 1)}

\section{Pembrolizumab}

In a phase Ib study from KEYNOTE-028 that explored the efficacy and safety of pembrolizumab (anti-PD-1 mAb) in late-line treatment ( $87 \%$ patients had received $\geq 2$ lines of treatment) for all histological types of EC (78\% ESCC, $22 \%$ EAC). Twenty-three patients had PD-L1-positive tumors. The mOS was 7 months (21). The phase II trial KEYNOTE-180 investigated pembrolizumab monotherapy in late-line $(\geq 3)$ therapy for patients with PD-L1-postive tumor. ESCC and EAC were both included. The mOS and median PFS (mPFS) were 5.8 and 2 months, respectively.
Subgroup analysis showed that the ORR and mOS were better in patients with ESCC (22). In the phase III trial from KEYNOTE-181, pembrolizumab used in the second or later line of therapy did not have significant survival benefit compared with chemotherapy. However, in the subgroup of patients with PD-L1 combined positive score (CPS) (PD-L1 expression on tumors and immune cells were both included) $\geq 10$, pembrolizumab treatment achieved a longer mOS than chemotherapy (9.3 vs. 6.7 months), and the ORR was also improved $(21.5 \%$ and $6.1 \%)$ in this subgroup (23).

\section{Nivolumab}

ATTRACTION-1, a Japanese phase II study, investigated the effect of nivolumab (anti-PD-1 mAb) on ESCC. The ORR was $17 \%$, and the mOS and mPFS were 10.8 and 1.5 months, respectively (24). ATTRACTION-03 (NCT02569242) enrolled patients with unresectable advanced or recurrent ESCC. The mOS was significantly improved in the nivolumab group compared with that in the chemotherapy group (10.9 vs. 8.4 months) (25). Meanwhile, the CheckMate-032 study (NCT01928394) assessed the efficacy and safety of nivolumab and nivolumab plus ipilimumab in three groups of EC patients (nivolumab $3 \mathrm{mg} / \mathrm{kg}$; nivolumab $1 \mathrm{mg} / \mathrm{kg}$ plus ipilimumab $3 \mathrm{mg} / \mathrm{kg}$; and nivolumab $3 \mathrm{mg} / \mathrm{kg}$ plus ipilimumab $1 \mathrm{mg} / \mathrm{kg}$ ). The ORR was $12 \%, 24 \%$, and $8 \%(95 \%$ CI, $2 \%$ to $19 \%)$ in the three groups, respectively (26). The RAMONA study, a multicenter, open-label phase II trial, has the primary objective of demonstrating the significant survival benefit of nivolumab and ipilimumab in older patients with advanced ESCC based on a comparison with historical data of standard chemotherapy; the study is ongoing (27).

\section{Camrelizumab}

One trial (NCT02742935), a dose-escalating phase I study, evaluated the efficacy and safety of camrelizumab (also name SHR-1210, humanized anti-PD1 IgG4 antibody developed by Jiangsu Hengrui Medicine Co. Ltd, China) as a second or later-line treatment for ESCC. The dose was given at 60, 200, and $400 \mathrm{mg}$ every 2 weeks. The ORR was $33.3 \%$ and the mPFS was 3.6 months (28). The ESCORT (NCT03099382) study to assess the efficacy and safety of camrelizumab $v s$. chemotherapy in patients with advanced or metastatic ESCC after the failure of first-line standard therapy, was the first randomized phase III study conducted 


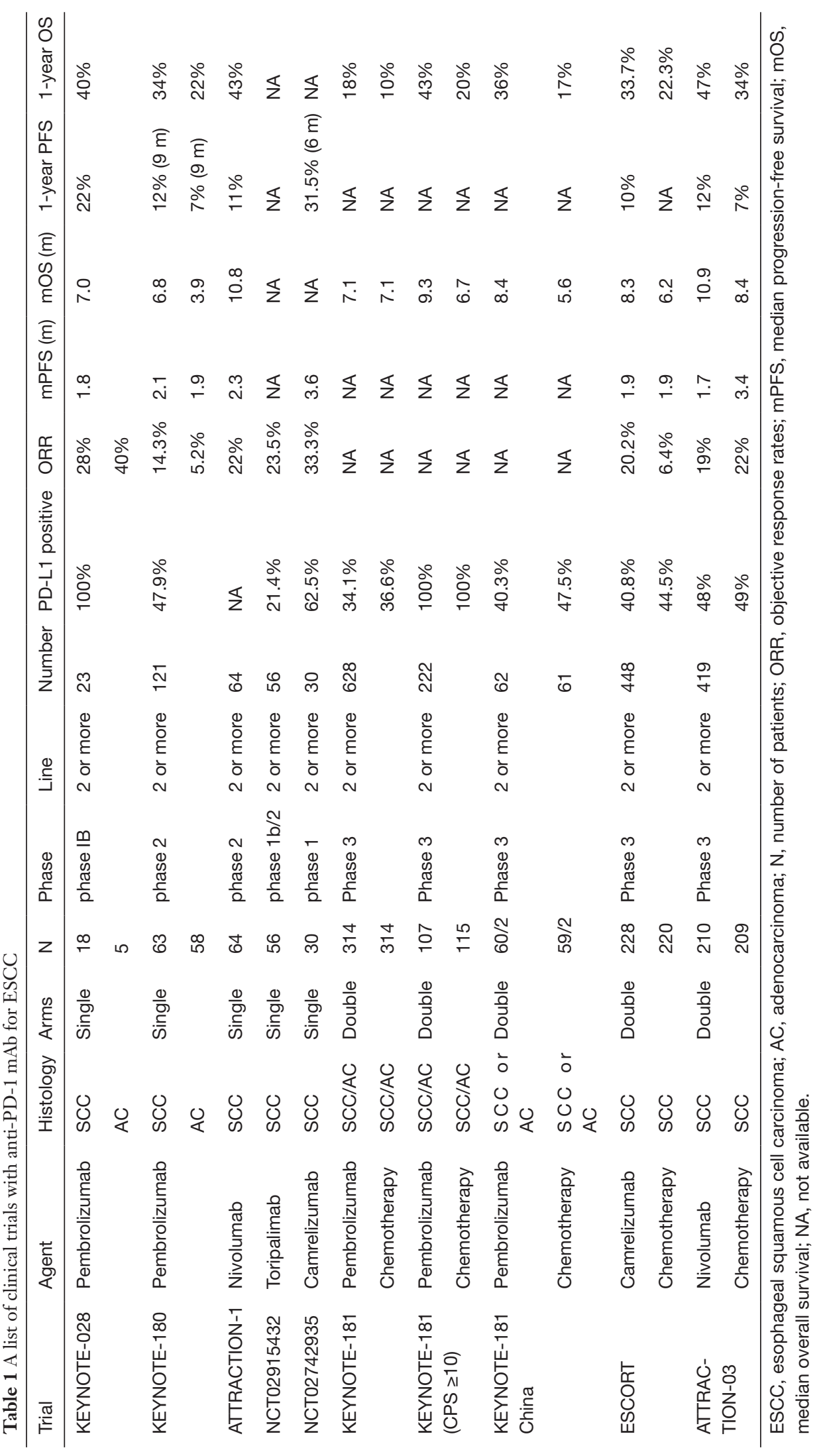


in a Chinese population. The results revealed improved mOS and a $31 \%$ reduction in the risk of progression or death after camrelizumab-treatment compared with after chemotherapy, as well as superior ORR (20.2\% vs. 6.4\%) and duration of response ( $7.4 v s .3 .4$ months).

\section{Toripalimab}

A phase Ib/II trial (NCT02915432) was conducted to evaluate the efficacy and safety of toripalimab (humanized anti-PD-1 mAb developed by Shanghai Junshi Bioscience) in refractory/metastatic ESCC. Of 34 patients involved in the trial, 8 achieved partial response, with an ORR of $23.5 \%(29)$.

\section{ICIs combined with chemotherapy in ESCC}

Two mechanisms are involved in chemotherapy-induced immune response. First, chemotherapeutic drugs influence the immune effect of tumor cells. The capacity of cyclophosphamide, gemcitabine, platinum, and paclitaxel to enhance the antigens of tumor cells has been shown (30). Paclitaxel, cisplatin, and doxorubicin could increase the immunogenicity of tumor cells by promoting immunogenic cell death of tumor cells and the expression and release of immunogenic substances from tumor cells, as well as by enhancing the immune effect sensitivity of tumor cells (31). Second, chemotherapeutic drugs can influence immune cells. Cytotoxic $\mathrm{T}$ lymphocytes are activated after treatment with Taxol, doxorubicin, or cisplatin. Taxol, gemcitabine, and 5 -fluorouracil can eliminate immunosuppressive cells. Changes in the tumor microenvironment, such as an increase in the release of tumor antigens, promote antigen cross-presentation, and thus accelerate anti-tumor immune response (31). The effects of chemotherapy on tumor cells and immune cells reciprocate, and re-shape the tumor immune response .

Several studies have demonstrated that immunotherapy combined with chemotherapy improved ORR and survival in multiple tumors and that the TRAEs are well tolerated. Since neoadjuvant chemotherapy has been shown to increase PD-L1 expression and CD8 ${ }^{+}$TILs in ESCC (32), chemotherapy combined with PD-1/PD-L1 blockade might achieve a potential clinical outcome. Although no reports currently exist, two randomized, double-blind, placebo-controlled, multicenter phase IIIclinical trials are under way. The first of these trials, KEYNOTE-590 (NCT03189719), will evaluate the efficacy and safety of cisplatin and 5-fluorouracil plus pembrolizumab vs. cisplatin and 5-fluorouracil plus placebo in patients with previously untreated advanced E/EGJ carcinoma (33). The second, SHR-1210-III-306 (NCT03691090), will compare the efficacy and safety of SHR-1210 + paclitaxel + cisplatin vs. placebo + paclitaxel + cisplatin as first-line therapy for patients in China with advanced EC.

\section{ICls combined with RT in ESCC}

The combination of ICIs with RT has drawn an increasing level of attention in recent years. RT boosts in situ vaccination by inducing immunogenic death in radiated tumor cells. RT reprograms the tumor immune microenvironment through the release of inflammatory cytokines and chemokines, which contributes to the recruitment of effector $T$ cells in tumors and converts the tumors into "inflamed" tissues which are susceptible to T-cell attack. Our data demonstrated an elevated expression of PD-L1 on ESCC cell lines after radiation (10). Therefore, when immune response was promoted by RT-induced tumor destruction, PD-L1expressing tumor cells were also selected. For patients who received esophagectomy after neoadjuvant CCRT, PDL1 staining was linked with lower complete pathologic response rate $\left[16 \%(3 / 18)\right.$ in $\mathrm{PD}-\mathrm{L}^{+}$patients vs. $31 \%$ (9/29) in PD-L1 ${ }^{-}$patients] (34). Preclinical and early clinical data have revealed the synergistic effect of RT and PD-1/ CTLA-4 blockade, which has led to an increase in interest in the combined use of these two treatment strategies (35). In our phase Ib clinical trial to evaluate the safety and effect of RT combined with SHR-1210 as a first-line treatment in locally advanced ESCC patients who were intolerant to CCRT, 19 patients were finally enrolled. The ORR was $73 \%$, and the mPFS and mOS were 11.7 and 16.9 months, respectively. At 12 months, the rates of OS and PFS were $63.2 \%$ and $47.4 \%$, respectively. The most common type of toxicity was cutaneous capillary hemangioma (15 in grade 1 and 2 in grade 3). Grade 3 TRAEs occurred in 9 patients experienced (31). One patient experienced grade 4 lymphopenia, which was not treated (36).

\section{ICls combined with CRT in ESCC}

A number of clinical trials are being conducted to investigate the efficacy of immunotherapy combined with neoadjuvant CRT in esophageal and gastric cancer or gastroesophageal junction adenocarcinoma (NCT03064490, NCT02730546, 
NCT02735239, NCT02962063, NCT03044613 and NCT03278626). The primary outcomes from these phase I/II clinical trials are safety and/or the rate of pathologic complete response as determined at the time of surgery. In addition to these trials, others are incorporating immunotherapy into the definitive, combined modality management of EC (NCT03377400 and NCT03544736).

In a phase II trial (NCTNCT02844075), patients with stage Ib to III ESCC received pembrolizumab combined with neoadjuvant CCRT over 5 weeks, followed by surgery, and for another two years after surgery. Of the 28 patients enrolled, 26 patients received esophagectomy, while 2 patients did not undergo surgery due to death (hematemesis) and the withdrawal of consent. After surgery, two patients died from acute lung injury. The pathologic complete response (pCR) in the primary tumor was $46.1 \%$. The 6- and 12-month OS rates were $89.3 \%$ and $82.1 \%$, respectively. The most common TRAEs in the neoadjuvant and adjuvant periods were neutropenia $(50.0 \%)$ and liver enzyme elevation (30.8\%), respectively (37). Another phase II clinical trial will evaluate the safety and efficacy of atezolizumab (anti-PD-L1 mAb) monotherapy following definitive CRT in patients with unresectable locally advanced ESCC. Within 4 weeks after CRT, the patients will receive atezolizumab every 3 weeks for 12 months or until disease progression. The trial will enroll a total of 50 patients including 40 with locally advanced primary ESCC and 10 with postoperative loco-regionally recurrent ESCC (38). Other than ESCC-focused studies, there is a phase II trial (NCT03087864) that is investigating neoadjuvant CRT combined with atezolizumab in resectable EAC. Of the 39 patients enrolled, 31 patients have completed neoadjuvant treatment and 8 patients are still receiving treatment. A pCR has been observed in 39\% of patients, which is promising compared to the $23 \%$ in the CROSS study in which neoadjuvant CRT was applied. Treatment-related immune AEs are manageable (39). The preliminary results from ongoing clinical trials have demonstrated that the addition of PD-1/PD-L1 blockade to preoperative CRT or definite CCRT has promising efficacy with manageable toxicity in EC. Based on the results, further investigation is warranted in a phase III clinical trial.

\section{Other combination treatment}

A single-center phase II study evaluated the efficacy and safety of camrelizumab plus apatinib, a tyrosine kinase inhibitor of vascular endothelial growth factor receptor-2 (VEGFR2-TKI), in combination with liposomal paclitaxel and nedaplatin in the first-line treatment of patients with ESCC. Response evaluation was available in 26 patients of 29 patients included. The ORR and DCR were $73.1 \%$ (19/26) and $96.2 \%$ (25/26), respectively. The most common grade $3 / 4$ adverse events were leucopenia $(21 / 29,72.4 \%)$ and neutropenia $(15 / 29,51.7 \%)$. The preliminary result indicated that ICIs plus VEGFR2-TKI in combination with chemotherapy might provide new treatment option for patients with unresectable locally advanced or metastatic $\operatorname{ESCC}(40)$.

\section{Treatment-related adverse effects}

A meta-analysis of 18,610 patients treated with PD-1 and PD-L1 inhibitors revealed that $66.0 \%$ patients developed at least $1 \mathrm{AE}$ of any grade, and $14.0 \%$ patients developed at least $1 \mathrm{AE}$ of grade 3 or higher severity (41). Different tumors appeared to have various TRAEs $(21,42)$. Several clinical trials have reported the TRAEs in ESCC patients treated with PD-1 or PD-L1 inhibitors (Table 2). TRAEs of any grade were less likely with pembrolizumab (39-65\%) and nivolumab (60-65\%) than with camrelizumab (83.394.3\%). The occurrence of grade 3-5 TRAEs was similar among pembrolizumab (12.4-18.2\%), nivolumab (17-18\%), and camrelizumab (10-19.3\%), which was lower than chemotherapy (39.5-63\%). The ongoing clinical trials have indicated that combining ICIs and chemotherapy or CCRT does not increase the occurrence of TRAEs compared with chemotherapy or CCRT.

TRAEs include diarrhea, decreased appetite, constipation, fatigue, and immune-related (irAEs), such as rash, hyperthyroidism, hypothyroidism, pneumonitis, and colitis. irAEs can be controlled by suspending the treatment or with corticosteroid treatment. It should be noted that there is a high incidence $(83.3 \%)$ of reactive capillary hemangiomas in camrelizumab, which are mostly of grade 1-2 and manageable with local therapy or observation. In order to prevent TRAEs, it is important to exclude patients who had serious baseline disease, such as in lung and digestive tract, before ICIs application. These patients are more susceptible to TRAEs. After ICIs application, the core principles of treating irAEs are early prevention and diagnosis as early as possible, and performing proper management. It is also necessary to monitor for the recurrence of irAEs when ICI treatment is resumed. 
Table 2 A list of treatment-related adverse events of anti-PD-1 mAb for ESCC

\begin{tabular}{|c|c|c|c|c|c|}
\hline Trial & Agent & TRAEs & Grade 3-5 TRAEs & Events leading to discontinuation & Events leading to death \\
\hline KEYNOTE-180 & Pembrolizumab & $57.9 \%$ & $12.4 \%$ & $4.1 \%$ & $0.9 \%$ \\
\hline ATTRACTION-1 & Nivolumab & $60 \%$ & $17 \%$ & $11 \%$ & 0 \\
\hline NCT02742935 & Camrelizumab & $83.3 \%$ & $10 \%$ & 0 & 0 \\
\hline KEYNOTE-181 & Chemotherapy & $86.1 \%$ & $40.7 \%$ & $6.4 \%$ & $1.7 \%$ \\
\hline \multirow[t]{2}{*}{ ESCORT } & Camrelizumab & $94.3 \%$ & $19.3 \%$ & $13.2 \%$ & $3.1 \%$ \\
\hline & Chemotherapy & $90.0 \%$ & $39.5 \%$ & $9.5 \%$ & $1.4 \%$ \\
\hline ATTRACTION-03 & Nivolumab & $65 \%$ & $18 \%$ & $9 \%$ & $0.96 \%$ \\
\hline
\end{tabular}

ESCC, esophageal squamous cell carcinoma; TRAEs, treatment-related adverse events.

\section{Biomarkers for predicting the efficacy of ICIs in ESCC (Table 3)}

The reported studies indicated the ORR of $10-30 \%$ in ESCC patients treated with ICIs, and there is still a large proportion of patients who do not benefit from ICI treatment. There is an urgent need for potential predictive biomarkers to select the most suitable patients, optimize therapeutic strategies, and monitor the clinical response. Unlike the precise targets and definite mechanism in targeted therapy, much more complicated factors arising from tumor cells and the tumor immune microenvironment harmonize and contribute to the clinical outcome of ICIbased treatment. A series of immune-related biomarkers, such as PD-L1 expression, TMB, MSI, and TILs, have been observed as valuable predictive candidates in ICIbased treatment for ESCC (43).

\section{$P D-L 1$}

An increasing level of evidence has shown that PDL1 expression in tumor cells is potential biomarker for predicting the therapeutic efficacy of $\mathrm{PD}-1 / \mathrm{PD}-\mathrm{L} 1$ inhibitors $(22,44,45)$. The level of PD-L1 expression can be assessed by tumor proportion score (TPS), which is defined as the tumor percentage of viable tumor cells showing partial or complete membrane staining and CPS. It is evaluated based on the total number of PD-L1 positive cells (tumor, lymphocytes, and macrophages) compared to total tumor cells; hence, the factors from both tumor and immune cells can be considered comprehensively. Several studies have suggested that patients with tumors of high CPS or TPS benefit from pembrolizumab treatment. In the KEYNOTE-180 trial, positive PD-L1 expression (defined as CPS $\geq 10$ ) in esophageal tumors was $47.9 \%$ (58/121). Patients with PD-L $1^{+}$expression had a higher 6-month PFS rate (22\% vs. 10\%) and 9-month PFS rate (14\% vs. $5 \%$ ) than those with $\mathrm{PD}-\mathrm{L}^{-}$expression (22). In a phase III KEYNOTE-181 study of pembrolizumab $v s$. the investigator's choice of chemotherapy as secondline therapy for patients with advanced/metastatic ESCC, EAC, or esophagogastric Junction (EGJ) (NCT02564263), pembrolizumab was superior to chemotherapy in terms of OS in patients with PD-L1 CPS $\geq 10$ (Hazard ratio 0.69; 95\% CI: 0.52-0.93; $\mathrm{P}=0.0074)$. The 12 -month OS rate in patients with CPS $\geq 10$ was $43 \%$ vs. $20 \%$ (23).

Some studies have also indicated a poor association between PD-L1 and clinical response. Huang et al. found that in 24 patients with metastatic ESCC, 15 (62.5\%) had $\mathrm{PD}-\mathrm{L}^{+}$tumors (defined as $\geq 5 \%$ ). The patients with PD$\mathrm{L}^{+}$tumors tended to have higher ORR (7 of 15 patients, $46.7 \%$ ) than in those with PD-L1 ${ }^{-}$tumors (1 of 9, $11.1 \%$ ), but there was no statistical difference $(\mathrm{P}>0.05)(28)$. A phase Ib/II study of JS001 (anti-PD-1 mAb developed by Shanghai Junshi Biosciences Co., Ltd, China) as salvage treatment for advanced ESCC showed 12 (21.4\%) of 56 patients had tumors with PD-L1 ${ }^{+}$expression (defined as positive staining $1 \%$ on tumor cells or on immune cells). Partial response was similar between $\mathrm{PD}-\mathrm{L}^{+}$patients $(20 \%$, 2/10) and PD-L1' patients (25\%, 6/24) (29). 
Table 3 A list of biomarkers in immune checkpoint inhibitor therapy for ESCC

\begin{tabular}{|c|c|c|c|c|c|}
\hline Factor & $\begin{array}{l}\text { Association with } \\
\text { favorable clinical } \\
\text { outcome }\end{array}$ & $\begin{array}{l}\text { Tissue type for biomarker } \\
\text { assessment }\end{array}$ & $\begin{array}{l}\text { Possible assay type for } \\
\text { biomarker assessment }\end{array}$ & Cutoff value (\%) & Positive rate (\%) \\
\hline $\begin{array}{l}\text { Tumor mutational } \\
\text { burden }\end{array}$ & High & Tissue/blood (ctDNA) & $\begin{array}{l}\text { WES or targeted } \\
\text { sequence }\end{array}$ & 7.7 & Media \\
\hline Copy number variation & High/low & Tissue & $\begin{array}{l}\text { WES or targeted } \\
\text { sequence }\end{array}$ & NA & NA \\
\hline $\begin{array}{l}\text { T cell repertoire clonality } \\
\text { change }\end{array}$ & Clonality/diversity & Tissue/blood & $\begin{array}{l}\text { Multiple PCR or } \\
\text { sequence }\end{array}$ & NA & NA \\
\hline
\end{tabular}

ESCC, esophageal squamous cell carcinoma; IHC, immunohistochemistry; CPS, combined positive score; MSI-H, high microsatellite instability; dMMR, deficient mismatch repair; PCR, pathologic complete response.

Of the various anti-PD-L1 antibodies available for testing PD-L1 expression, four antibodies (22C3 and 28-8 from Dako; SP263 and SP142 from Ventana) have been used widely in clinical trials. Despite these antibodies recognizing different peptide-recognizing domains in PDL1 antigen, their testing efficacy has been shown to be consistent apart from a relatively lower positive detection rate in SP142. Because of antigen degradation during preservation and heterogeneous expression in tumor tissues across different locations, the preservation time and specimen size (surgery $v$ s. biopsy) should also be considered when PD-L1 expression is evaluated.

\section{$T M B$}

There is a significant correlation between the TMB and the response to PD-1/PD-L1 inhibitors in a wide variety of cancers (17). EC has an upper middle level of TMB in solid tumors $(46,47)$. Huang et al. performed exome sequencing on the DNAs from paired tumors and white blood cells from 23 patients with metastatic ESCC (28). The mutation count per tumor ranged from 15 to 219 , with a median of 60 mutations per tumor. Using 60 mutations per tumor as the cut-off value, 8 of 11 samples with high TMB showed clinical benefit and only 2 of the 12 samples with low TMB had clinical benefit $(\mathrm{P}=0.0123)$. Further explorations of TMB in EC treated with immunotherapy are ongoing. Although TMB is an important feature correlated with immunotherapy, it is time-consuming and requires a higher quality of specimens compared with IHC-based PD-L1 assessment. TMB will not replace PD-L1 test, but could be a beneficial supplement.

\section{MSI}

DNA replication errors are checked and corrected by MMR system. MMR proteins including MLH1, PMS2, MSH2, and MSH6 can form heterodimers to correct such mismatches. Deficient mismatch repair (dMMR) in tumor cells causes areas of DNA to become unstable, resulting in MSI. Tumors with a high level of MSI (MSI-H) are likely to have a high TMB count and an increased number of tumor neoantigens. Thus, powerful local and systemic immune responses might be induced (48). Chalmers et al. analyzed MSI-H and TMB in 62,150 patients with solid tumors and found that the vast majority of MSI-H samples also had high TMB (83\%) and $97 \%$ had TMB $\geq 10$ mutations/Mb (46). A clinical trial of PD-1 blockade in patients with advanced MMR-deficient in 12 different types of tumor showed that $53 \%$ and $21 \%$ of patients achieved objective radiographic response or complete response, respectively (49). Other results from 5 clinical trials including 15 tumor entities with MSI/dMMR tumors (KEYNOTE-012, -016, -028, -158 and -164) also revealed a high correlation between MSI and a better response to ICIs. These findings led the FDA to approve a tumor-type agnostic pembrolizumab for all 
microsatellite-unstable tumors.

The dMMR in esophageal carcinoma stands at $4.35 \%$ (47). In the KEYNOTE-012 study, 24 samples of gastric or EGJ cancer were analyzed, and $17 \%$ of which were MSI-H tumors. In the CHECKMATE-032 study, in which patients with esophageal, EGJ, or gastric cancer were included, MSI-H occurred in $18 \%$ of samples (26). MSI is rarely reported in ESCC and is a potential area of focus for future studies.

\section{T-cell-inflamed gene-expression profile (GEP)}

The ability of the host immune system to recognize and eliminate tumor cells is orchestrated by innate and adaptive antitumor immune response. IFN- $\gamma$ is the key cytokine and plays a vital role in the antitumor response. Meanwhile, as a feedback mechanism, IFN- $\gamma$ signaling activates the PD-1 signaling axis via the upregulation of PD-L1 and PD-L2 in tumor cells as well as in stromal cells. Multigene immune signatures might comprehensively capture a complex, $\mathrm{T}$ cell-inflamed phenotype necessary for the clinical activity of PD-1/PD-L1 inhibitors.

In a pilot study involving 19 melanoma patients and, eventually, 220 patients with 9 cancers from KEYNOTE-012 and -028, a T cell-inflamed GEP comprising 18 genes was found to be associated with response to pembrolizumab, independently based on PDL1 expression (50). The GFP genes selected were related to antigen presentation, chemokine expression, cytotoxic activity, and adaptive immune resistance, and were identical to the 18 genes in the tumor inflammation signature $(50,51)$. Later, the KEYNOTE-028 study of 313 patients consistently found higher T-cell-inflamed GFP score in patients who achieved improved ORR and longer PFS after pembrolizumab treatment (44). In addition to these two studies, an analysis of 23 patients with EC (including 8 patients with ESCC) from KEYNOTE-028 used an IFN- $\gamma$ GFP signature that included 6 genes of the 18-gene profile. The result also suggested that the patients with higher IFN- $\gamma$ composite scores achieved better treatment outcomes (21).

\section{TILs}

During tumor progression and the application of various therapies, lymphocytes migrate to the tumor tissues to serve as effectors of antitumor response. At the same time, because of the inhibitory tumor microenvironment or feedback mechanism, TILs are usually inactivated. Since ICIs target inhibitory checkpoint molecules, antitumor response might be reactivated in tumors with high levels of TILs. An early study of metastatic melanoma suggested that TILs were predictive of response in both CTLA-4 and PD-1 blockade. TILs were found at a higher density early during treatment in patients who showed a better response (52). A study examined the prognostic impact of PD-L1 expression and TIL status, and their combination, in EC. Of the 305 patients included, 279 (91\%) had ESCC. The results indicated that both PD-L1 positivity and TIL positivity were significantly correlated with better OS and DFS. There was also a close association observed between TIL positivity and high expression of CD8. Interestingly, the combination of PD-L1 expression and TIL status could further classify the patients according to clinical outcome: the patients in the PD-L1- $\mathrm{TIL}^{+}$group had the longest OS and DFS, and those in the PD-L1 ${ }^{+} \mathrm{TIL}^{-}$group had the shortest OS and DFS (53). Patients with PD-L1'TIL ${ }^{+}$ tumors were the most suitable candidates to receive monoPD-1/PD-L1 inhibitors. Patients with PD-L1'- TIL $^{+}$tumors would not benefit from PD-1/PD-L1 blockade. For patients with either PD-L1 ${ }^{-} \mathrm{TIL}^{-}$or PD-L1 ${ }^{+} \mathrm{TIL}^{-}$tumors, strategies to attract TILs are needed first before any ICI treatment is performed (53).

\section{Other biomarkers}

A recent study in advanced melanoma indicated that the functional immunological assay on ex vivo tumormetastatic lymph nodes could serve as a new predictor in immunotherapy (54). Circulating immune cells, which are more convenient and can be obtained less invasively than tumor tissue, are potential biomarkers for predicting treatment response to ICIs. PD-L1 expression on peripheral T cells was associated with the response to anti-CTLA-4 $\mathrm{mAb}$ (54). Meanwhile, CD137 expression on circulating $\mathrm{CD}^{+} \mathrm{T}$ cells was discovered to be a promising predictor after adjuvant ipilimumab combined with nivolumab (54). Circulating tumor DNA (ctDNA) after CRT has been shown to be associated with tumor progression, metastasis, and disease-specific survival in patients with EC (55). Improved antitumor immunity and response were found in melanoma patients with favorable gut microbiomes (55). Moreover, another study showed that gut microbiome promoted the efficacy of ICIs through the recruitment 
of $\mathrm{T}$ cells into the tumor beds (56). Although the role of esophageal microbiomes in ESCC patients is not well known, a study including 25 ESCC patients and 50 matched controls found that Prevotella, especially Prevotella nanceiensis, was abundant in patients with ESCC (57). Furthermore, another case-control study on 210 esophageal samples from 86 patients suggested that there was decreased microbial diversity in EAC tissues compared with tissues from healthy control patients (58). The association between the esophageal microbiome and the treatment response to ICIs requires further study in future investigations.

Because of intratumoral heterogeneity, the levels of biomarkers from biopsies obtained from different sites might be inconsistent in a single patient. The fluctuating levels of biomarkers may also be attributed to individual genetic background. Different therapeutic strategies and alterations of the tumor microenvironment and systematic immunity during treatment result in dynamic changes in some predictive biomarkers, such as PD-L1 expression, TILs, and peripheral immune cells. Furthermore, a lack of consistency between different biomarkers might be explained by the different mechanisms involved (59). In TMB, specific mutations in oncogenes and tumor PD-L1 expression describe the tumor component while immunecell PD-1/PD-L1 expression, HLA genotype, TCR repertoire, and possibly immune signatures represent the antitumor immune response. Therefore, a combination of several biomarkers with different mechanism could achieve enhanced ability to predict the clinical response and treatment outcome.

\section{Conclusions and future directions}

The findings of clinical trials have emphasized that the combination of ICIs with chemotherapy or RT might achieve greater treatment efficacy for various cancers compared to the use of ICIs alone. The studies of antiPD-1/L1 antibody combined with CRT in ESCC are ongoing. The exploration of strong predictive and prognostic biomarkers or integrated biomarkers to optimize therapeutic strategies is another area of focus for research. Meanwhile, the identification of valuable biomarkers for predicting toxicity is also crucial. With a deepening understanding of the biological tumor cells and antitumor immune features in ESCC, it is reasonable to believe that ICIs, or their application in combined treatment, will benefit more patients in future.

\section{Acknowledgments}

Funding: This work was supported by the National Nature Science Foundation of China (Grant No. 81872462).

\section{Footnote}

Reporting Checklist: The authors have completed the Narrative Review reporting checklist. Available at http:// dx.doi.org/10.21037/atm-20-4625

Conflicts of Interest: All authors have completed the ICMJE uniform disclosure form (available at http://dx.doi. org/10.21037/atm-20-4625). The authors have no conflicts of interest to declare.

Ethical Statement: The authors are accountable for all aspects of the work in ensuring that questions related to the accuracy or integrity of any part of the work are appropriately investigated and resolved.

Open Access Statement: This is an Open Access article distributed in accordance with the Creative Commons Attribution-NonCommercial-NoDerivs 4.0 International License (CC BY-NC-ND 4.0), which permits the noncommercial replication and distribution of the article with the strict proviso that no changes or edits are made and the original work is properly cited (including links to both the formal publication through the relevant DOI and the license). See: https://creativecommons.org/licenses/by-nc-nd/4.0/.

\section{References}

1. Bray F, Ferlay J, Soerjomataram I, et al. Global cancer statistics 2018: GLOBOCAN estimates of incidence and mortality worldwide for 36 cancers in 185 countries. CA Cancer J Clin 2018;68:394-424.

2. Cancer Genome Atlas Research N, Analysis Working Group: Asan U, Agency BCC, et al. Integrated genomic characterization of oesophageal carcinoma. Nature 2017;541:169-75.

3. Yang H, Liu H, Chen Y, et al. Neoadjuvant Chemoradiotherapy Followed by Surgery Versus Surgery Alone for Locally Advanced Squamous Cell Carcinoma of the Esophagus (NEOCRTEC5010): A Phase III Multicenter, Randomized, Open-Label Clinical Trial. J Clin Oncol 2018;36:2796-803.

4. Shapiro J, van Lanschot JJB, Hulshof M, vet al. 
Neoadjuvant chemoradiotherapy plus surgery versus surgery alone for oesophageal or junctional cancer (CROSS): long-term results of a randomised controlled trial. Lancet Oncol 2015;16:1090-8.

5. Li H, Fang W, Yu Z, et al. Chinese expert consensus on mediastinal lymph node dissection in esophagectomy for esophageal cancer (2017 edition). J Thorac Dis 2018;10:2481-9.

6. Suntharalingam M, Winter K, Ilson D, et al. Effect of the Addition of Cetuximab to Paclitaxel, Cisplatin, and Radiation Therapy for Patients With Esophageal Cancer: The NRG Oncology RTOG 0436 Phase 3 Randomized Clinical Trial. JAMA Oncol 2017;3:1520-8.

7. Jiao R, Luo $\mathrm{H}, \mathrm{Xu} \mathrm{W}$, et al. Immune checkpoint inhibitors in esophageal squamous cell carcinoma: progress and opportunities. Onco Targets Ther 2019;12:6023-32.

8. Li X, Shao C, Shi Y, et al. Lessons learned from the blockade of immune checkpoints in cancer immunotherapy. J Hematol Oncol 2018;11:31.

9. Pardoll DM. The blockade of immune checkpoints in cancer immunotherapy. Nat Rev Cancer 2012;12:252-64.

10. Zhang W, Pang Q, Zhang X, et al. Programmed deathligand 1 is prognostic factor in esophageal squamous cell carcinoma and is associated with epidermal growth factor receptor. Cancer Sci 2017;108:590-7.

11. Ohigashi $Y$, Sho M, Yamada $Y$, et al. Clinical significance of programmed death-1 ligand-1 and programmed death-1 ligand-2 expression in human esophageal cancer. Clin Cancer Res 2005;11:2947-53.

12. Qu HX, Zhao LP, Zhan SH, et al. Clinicopathological and prognostic significance of programmed cell death ligand 1 (PD-L1) expression in patients with esophageal squamous cell carcinoma: a meta-analysis. J Thorac Dis 2016;8:3197-204.

13. Tsutsumi S, Saeki H, Nakashima Y, et al. Programmed death-ligand 1 expression at tumor invasive front is associated with epithelial-mesenchymal transition and poor prognosis in esophageal squamous cell carcinoma. Cancer Sci 2017;108:1119-27.

14. Huang WT, Lu HI, Wang YM, et al. Positive Programmed Cell Death-Ligand 1 Expression Predicts Poor Treatment Outcomes in Esophageal Squamous Cell Carcinoma Patients Receiving Neoadjuvant Chemoradiotherapy. J Clin Med 2019;8.

15. Vogelstein B, Papadopoulos N, Velculescu VE, et al. Cancer genome landscapes. Science 2013;339:1546-58.

16. Alexandrov LB, Nik-Zainal S, Wedge DC, et al. Signatures of mutational processes in human cancer. Nature
2013;500:415-21.

17. Yarchoan M, Hopkins A, Jaffee EM. Tumor Mutational Burden and Response Rate to PD-1 Inhibition. N Engl J Med 2017;377:2500-1.

18. Zhou S, Yang H, Zhang J, et al. Changes in indoleamine 2,3-dioxygenase 1 expression and CD8+ tumor-infiltrating lymphocytes after neoadjuvant chemoradiotherapy and prognostic significance in esophageal squamous cell carcinoma. Int J Radiat Oncol Biol Phys 2020;S03603016(20)30126-7.

19. Qian D, Wang Y, Zhao G, et al. Tumor Remission and Tumor-Infiltrating Lymphocytes During Chemoradiation Therapy: Predictive and Prognostic Markers in Locally Advanced Esophageal Squamous Cell Carcinoma. Int J Radiat Oncol Biol Phys 2019;105:319-28.

20. Hatogai K, Fujii S, Kitano S, et al. Relationship between the immune microenvironment of different locations in a primary tumour and clinical outcomes of oesophageal squamous cell carcinoma. Br J Cancer 2020;122:413-20.

21. Doi T, Piha-Paul SA, Jalal SI, et al. Safety and Antitumor Activity of the Anti-Programmed Death-1 Antibody Pembrolizumab in Patients With Advanced Esophageal Carcinoma. J Clin Oncol 2018;36:61-7.

22. Shah MA, Kojima T, Hochhauser D, et al. Efficacy and Safety of Pembrolizumab for Heavily Pretreated Patients With Advanced, Metastatic Adenocarcinoma or Squamous Cell Carcinoma of the Esophagus: The Phase 2 KEYNOTE-180 Study. JAMA Oncol 2019;5:546-50.

23. Kojima T, Muro K, Francois E, et al. Pembrolizumab versus chemotherapy as second-line therapy for advanced esophageal cancer: Phase III KEYNOTE-181 study. J Clin Oncol 2019;37:2.

24. Kudo T, Hamamoto Y, Kato K, et al. Nivolumab treatment for oesophageal squamous-cell carcinoma: an open-label, multicentre, phase 2 trial. Lancet Oncol 2017;18:631-9.

25. Kato K, Cho BC, Takahashi M, et al. Nivolumab versus chemotherapy in patients with advanced oesophageal squamous cell carcinoma refractory or intolerant to previous chemotherapy (ATTRACTION-3): a multicentre, randomised, open-label, phase 3 trial. Lancet Oncol 2019;20:1506-17.

26. Janjigian YY, Bendell J, Calvo E, et al. CheckMate-032 Study: Efficacy and Safety of Nivolumab and Nivolumab Plus Ipilimumab in Patients With Metastatic Esophagogastric Cancer. J Clin Oncol 2018;36:2836-44.

27. Meindl-Beinker NM, Betge J, Gutting T, et al. A multicenter open-label phase II trial to evaluate nivolumab and ipilimumab for 2nd line therapy in elderly 
patients with advanced esophageal squamous cell cancer (RAMONA). BMC Cancer 2019;19:231.

28. Huang J, Xu B, Mo H, et al. Safety, Activity, and Biomarkers of SHR-1210, an Anti-PD-1 Antibody, for Patients with Advanced Esophageal Carcinoma. Clin Cancer Res 2018;24:1296-304.

29. Xu RH, Wang F, Shi J, et al. Recombinant humanized antiPD-1 monoclonal antibody (JS001) as salvage treatment for advanced esophageal squamous cell carcinoma: Preliminary results of an open-label, multi-cohort, phase Ib/II clinical study. J Clin Oncol 2018;36:116.

30. Zitvogel L, Galluzzi L, Smyth MJ, et al. Mechanism of action of conventional and targeted anticancer therapies: reinstating immunosurveillance. Immunity 2013;39:74-88.

31. Galluzzi L, Buque A, Kepp O, et al. Immunological Effects of Conventional Chemotherapy and Targeted Anticancer Agents. Cancer Cell 2015;28:690-714.

32. Fukuoka E, Yamashita K, Tanaka T, et al. Neoadjuvant Chemotherapy Increases PD-L1 Expression and CD8(+) Tumor-infiltrating Lymphocytes in Esophageal Squamous Cell Carcinoma. Anticancer Res 2019;39:4539-48.

33. Kato K, Shah MA, Enzinger P, et al. KEYNOTE-590: Phase III study of first-line chemotherapy with or without pembrolizumab for advanced esophageal cancer. Future Oncol 2019;15:1057-66.

34. Chen MF, Chen PT, Chen WC, et al. The role of PD$\mathrm{L} 1$ in the radiation response and prognosis for esophageal squamous cell carcinoma related to IL-6 and T-cell immunosuppression. Oncotarget 2016;7:7913-24.

35. Twyman-Saint Victor C, Rech AJ, Maity A, et al. Radiation and dual checkpoint blockade activate non-redundant immune mechanisms in cancer. Nature 2015;520:373-7.

36. Pang Q, Li X, Zhang W, et al. Safety and Effect of Radiation Therapy Combined with Anti-PD-1 Antibody SHR-1210 as First-Line Treatment on Patients with Intolerable Concurrent Chemoradiotherapy Esophageal Cancer: A Phase 1B Clinical Trial. Int J Radiat Oncol Biol Phys 2018;102:e39.

37. Hong MH, Kim H, Park SY, et al. A phase II trial of preoperative chemoradiotherapy and pembrolizumab for locally advanced esophageal squamous cell carcinoma (ESCC). J Clin Oncol 2019;37:4027.

38. Bando H, Kotani D, Tsushima T, et al. TENERGY: Multicenter phase II study of atezolizumab monotherapy following definitive chemoradiotherapy with 5-FU plus cisplatin in patients with locally advanced esophageal squamous cell carcinoma. J Clin Oncol 2019;37:TPS4141.

39. Ende T, Clercq NC, Henegouwen MI, et al. A phase
II feasibility trial of neoadjuvant chemoradiotherapy combined with atezolizumab for resectable esophageal adenocarcinoma: The PERFECT trial. J Clin Oncol 2019;37:4045.

40. Zhang B, Qi L, Wang X, et al. Phase 2 study of camrelizumab (anti-PD-1 antibody) combined with apatinib and chemotherapy for the first-line treatment of advanced esophageal squamous cell carcinoma. J Clin Oncol 2019;37:4033.

41. Wang Y, Zhou S, Yang F, et al. Treatment-Related Adverse Events of PD-1 and PD-L1 Inhibitors in Clinical Trials: A Systematic Review and Meta-analysis. JAMA Oncol 2019;5:1008-19.

42. Cho J, Kim HS, Ku BM, et al. Pembrolizumab for Patients With Refractory or Relapsed Thymic Epithelial Tumor: An Open-Label Phase II Trial. J Clin Oncol 2019;37:2162-70.

43. Wu HX, Wang ZX, Zhao Q, et al. Tumor mutational and indel burden: a systematic pan-cancer evaluation as prognostic biomarkers. Ann Transl Med 2019;7:640.

44. Ott PA, Bang YJ, Piha-Paul SA, et al. T-Cell-Inflamed Gene-Expression Profile, Programmed Death Ligand 1 Expression, and Tumor Mutational Burden Predict Efficacy in Patients Treated With Pembrolizumab Across 20 Cancers: KEYNOTE-028. J Clin Oncol 2019;37:318-27.

45. Fehrenbacher L, Spira A, Ballinger M, et al. Atezolizumab versus docetaxel for patients with previously treated non-small-cell lung cancer (POPLAR): a multicentre, open-label, phase 2 randomised controlled trial. Lancet 2016;387:1837-46.

46. Chalmers ZR, Connelly CF, Fabrizio D, et al. Analysis of 100,000 human cancer genomes reveals the landscape of tumor mutational burden. Genome Med 2017;9:34.

47. Zang YS, Dai C, Xu X, et al. Comprehensive analysis of potential immunotherapy genomic biomarkers in 1000 Chinese patients with cancer. Cancer Med 2019;8:4699-708.

48. Kloor M, Michel S, von Knebel Doeberitz M. Immune evasion of microsatellite unstable colorectal cancers. Int J Cancer 2010;127:1001-10.

49. Le DT, Durham JN, Smith KN, et al. Mismatch repair deficiency predicts response of solid tumors to PD-1 blockade. Science 2017;357:409-13.

50. Ayers M, Lunceford J, Nebozhyn M, et al. IFN-gammarelated mRNA profile predicts clinical response to $\mathrm{PD}-1$ blockade. J Clin Invest 2017;127:2930-40.

51. Danaher P, Warren S, Lu R, et al. Pan-cancer adaptive 
immune resistance as defined by the Tumor Inflammation Signature (TIS): results from The Cancer Genome Atlas (TCGA). J Immunother Cancer 2018;6:63.

52. Chen PL, Roh W, Reuben A, et al. Analysis of Immune Signatures in Longitudinal Tumor Samples Yields Insight into Biomarkers of Response and Mechanisms of Resistance to Immune Checkpoint Blockade. Cancer Discov 2016;6:827-37.

53. Yagi T, Baba Y, Ishimoto T, et al. PD-L1 Expression, Tumor-infiltrating Lymphocytes, and Clinical Outcome in Patients With Surgically Resected Esophageal Cancer. Ann Surg 2019;269:471-8.

54. Jacquelot N, Roberti MP, Enot DP, et al. Predictors of responses to immune checkpoint blockade in advanced melanoma. Nat Commun 2017;8:592.

55. Azad TD, Chaudhuri AA, Fang P, et al. Circulating Tumor
DNA Analysis for Detection of Minimal Residual Disease After Chemoradiotherapy for Localized Esophageal Cancer. Gastroenterology 2020;158:494-505.e6.

56. Routy B, Le Chatelier E, Derosa L, et al. Gut microbiome influences efficacy of PD-1-based immunotherapy against epithelial tumors. Science 2018;359:91-7.

57. Peters BA, Wu J, Pei Z, et al. Oral Microbiome Composition Reflects Prospective Risk for Esophageal Cancers. Cancer Res 2017;77:6777-87.

58. Elliott DRF, Walker AW, O'Donovan M, et al. A nonendoscopic device to sample the oesophageal microbiota: a case-control study. Lancet Gastroenterol Hepatol 2017;2:32-42.

59. Cristescu R, Mogg R, Ayers M, et al. Pan-tumor genomic biomarkers for PD-1 checkpoint blockade-based immunotherapy. Science 2018;362.
Cite this article as: Zhang W, Wang P, Pang Q. Immune checkpoint inhibitors for esophageal squamous cell carcinoma: a narrative review. Ann Transl Med 2020;8(18):1193. doi: 10.21037/atm-20-4625 